551. 593. $53: 551.521 .63$

\title{
Studies of the Distribution of Ultraviolet Sky Radiation (VII)
}

-On the Role of the Scattered Light Higher than the Second-

\author{
by \\ K. Sekihara \\ Meteorological Research Institute \\ (Received July 20, 1955)
}

\begin{abstract}
Intensities of light from the sky ranging from about $\lambda 4660 \mathrm{~A}^{\circ}$ to $\lambda 3115 \AA$ were calculated, using CHANDRASEKHAR's theory of radiative equilibrium in a RAYLEIGH atmosphere and the results were compared with observed distribution and also with theoretical results of direct integration of primary and secondary scattering. With the exception of the distribution when the sun's altitude is high where non-Rayleigh scattering is remarkable, the observed distributions were almost quantitatively explained by the theory of radiative equilibrium, but the theory of direct integration could not, because the scattered light of higher orders than the second, amounting to $30 \sim 40 \%$ in the range of the wave-length of about $\lambda 3115 \AA$, gave an essential feature to the distribution. However in the visible range these quantities wave at most several percent and the validity of the method of direct integration was confirmed to some extent.
\end{abstract}

\section{Introduction}

Measurement of the intensities of ultraviolet sky radiation was so far carried out in Europe partly from bioclimatological interest but its analysis from a purely geophysical view point was very rare and unsatisfactory until now. Chief difficulties in this study seem to have been in the evaluation of the multiply scattered light.

The theory of scattered sky radiation has made remarkable progress in these ten years or so, and at present two different methods of calculation are known to exist, the one being the method of direct integration including only primary and secondary scattering, which was chiefly developed by CHAPMAN and HAMmad [1], HAMMAD [2], [3] and this author [4], and the other being the method of radiative equilibrium including all orders of scattering, which was exclusively developed by Chandrasekhar [5], [6]. The latter method is now considered to be the more effective and was applied to the theory of sky light polarization by CHANDRASEKHAR himself[7], [8], to the calculation of night sky light by AshBurn[9], to the calculation concerning the observation of a star and also to the calculation of global radiation by Deirmendian and Sekera[10], [11]. But the method of direct integration also gives a satisfactory result at least in the visible range, and HAMMAD gave a satisfactory explanation on the sky light pelarization by this method and furthermore reached better agreement than CHANDRASEKHAR with the observed degree of 
polarization at the zenith by taking into account the anisotropy of air molecules.

The chief object of this study is to attain quantitative explanation on the angular distribution of ultraviolet sky radiation by means of CHANDRASEKHAR's theory but there is one more object, that is, to see the effect of scattered light higher than the second and clarify the limit of the availability of direct integration through the comparison of the results obtained by the above-mentioned two methods.

\section{The theory of radiative equilibrium in a Rayleigh atmosphere}

This theory which was developed by CHANDRASEKHAR during the years 1944 1948 in the Astrophysical Journal and described in detail in his book entitled "Radiative Transfer" (1950), is now well admitted to offer the most powerful weapons for the study of scattered sky radiation. And as it seems unneccessary to repeat here the details of the theory, only the essential parts will be presented below.

One of the great merits of Chandrasekhar is the rediscovery of Stokes' expression of a partially polarized light beam which enabled the basic equation of transfer to deal with. According to this mode of expression, a light beam can be represented by three parameters, that is, $I_{l}$, the intensity in a certain direction $l$ perpendicular to the direction of propagation, $I_{r}$ the intensity in the direction $r$ perpendicular to $l$ and also to the direction of propagation and $U$ determining the plane of polarization through the equation $\tan 2 \chi=U /\left(I_{l}-I_{r}\right)$ where $\chi$ is the angle between the plane of polarization and the direction $l$. Now, using these three STokes parameters, the intensity in the direction $\psi$ is expressed as

$$
I(\phi)=I_{l} \cos ^{2} \phi+I_{r} \sin ^{2} \phi+-\frac{1}{2} U \sin 2 \psi .
$$

Here to compose two independent light beams is merely to add the three parameters respectively and the scattering phenomena can be expressed as a linear transformation of the SToKes parameters. Thus the basic equation of transfer in a plane parallel atmosphere on RAYLEIGH scattering can be written as a vector equation in the following way:*

$$
\begin{aligned}
\mu \frac{d I(\tau, \mu, \varphi)}{d \tau}=\boldsymbol{I}(\tau, \mu, \varphi) & -\frac{1}{4 \pi} \int_{-1}^{+1} \int_{0}^{2 \pi} \boldsymbol{P}\left(\mu, \varphi, \mu^{\prime}, \varphi^{\prime}\right) \boldsymbol{I}\left(\tau, \mu^{\prime}, \varphi^{\prime}\right) d \mu^{\prime} d \varphi^{\prime} \\
& -\frac{1}{4} e^{-\tau / \mu_{0}} \boldsymbol{P}\left(\mu, \varphi ;-\mu_{0}, \varphi_{0}\right) \boldsymbol{F},
\end{aligned}
$$

where $\quad \tau$ : denotes the optical depth

$\mu:$ the cosine of zenith angles

$\varphi:$ the azimuthal angle measured from the direction of the sun

$\boldsymbol{I}=\left(I_{l}, I_{r}, U\right)$ : the Stokes vector of a light beam at the position concerned $\pi \boldsymbol{F}=\left(\pi F_{l}, \pi F_{r}, \pi F_{U}\right):$ the Stokes vector of an incident flux of parallel light beam

* Where $l$ and $r$ from now on refer to the directions parallel and perpendicular, respectively, to the meridian and in the plane containing the directions of the beam and of the normal to the plane of the stratification of the atmosphere. 
$\mu_{0}, \varphi_{0}$ : refers to the direction of $F$ respectively

$P$ : the scattering matrix formulated as follows:

$$
\begin{gathered}
\boldsymbol{P}\left(\mu, \varphi ; \mu^{\prime}, \varphi^{\prime}\right)=\boldsymbol{Q}\left[\boldsymbol{P}^{(0)}\left(\mu, \mu^{\prime}\right)+\left(1-\mu^{2}\right)^{\frac{1}{2}}\left(1-\mu^{\prime 2}\right)^{\frac{1}{2}} \boldsymbol{P}^{(1)}\left(\mu, \varphi ; \mu^{\prime}, \varphi^{\prime}\right)\right. \\
\left.+\boldsymbol{P}^{(2)}\left(\mu, \varphi ; \mu^{\prime}, \varphi^{\prime}\right)\right],
\end{gathered}
$$

where

$$
\begin{aligned}
& \boldsymbol{Q}=\left(\begin{array}{lll}
1 & 0 & 0 \\
0 & 1 & 0 \\
0 & 0 & 2
\end{array}\right) \\
& \boldsymbol{P}^{(0)}\left(\mu, \mu^{\prime}\right)=\frac{3}{4}\left(\begin{array}{ccc}
2\left(1-\mu^{2}\right)\left(1-\mu^{\prime 2}\right)+\mu^{2} \mu^{\prime 2} & \mu^{2} & 0 \\
\mu^{\prime 2} & 1 & 0 \\
0 & 0 & 0
\end{array}\right), \\
& \boldsymbol{P}^{(1)}\left(\mu, \varphi ; \mu^{\prime}, \varphi^{\prime}\right)=-\frac{3}{4}\left(\begin{array}{ccc}
4 \mu \mu^{\prime} \cos \left(\varphi^{\prime}-\varphi\right) & 0 & 2 \mu \sin \left(\varphi^{\prime}-\varphi\right) \\
0 & 0 & 0 \\
-2 \mu^{\prime} \sin \left(\varphi^{\prime}-\varphi\right) & 0 & \cos \left(\varphi^{\prime}-\varphi\right)
\end{array}\right) \text {, } \\
& \boldsymbol{P}^{(2)}\left(\mu, \varphi ; \mu^{\prime}, \varphi^{\prime}\right)=\frac{3}{4}\left(\begin{array}{ccc}
\mu^{2} \mu^{\prime 2} \cos 2\left(\varphi^{\prime}-\varphi\right) & -\mu^{2} \cos 2\left(\varphi^{\prime}-\varphi\right) & \mu^{2} \mu^{\prime} \sin 2\left(\varphi^{\prime}-\varphi\right) \\
-\mu^{\prime 2} \cos 2\left(\varphi^{\prime}-\varphi\right) & \cos 2\left(\varphi^{\prime}-\varphi\right) & -\mu^{\prime} \sin 2\left(\varphi^{\prime}-\varphi\right) \\
-\mu \mu^{\prime 2} \sin 2\left(\varphi^{\prime}-\varphi\right) & \mu \sin 2\left(\varphi^{\prime}-\varphi\right) & \mu \mu^{\prime} \cos 2\left(\varphi^{\prime}-\varphi\right)
\end{array}\right)
\end{aligned}
$$

The boundary conditions to be satisfied are

$$
\begin{array}{ll}
\boldsymbol{I}(0,-\mu, \varphi) \equiv 0 & (0<\mu \leq 1,0 \leq \varphi \leq 2 \pi) \\
\boldsymbol{I}\left(\tau_{1},+\mu, \varphi\right) \equiv 0 & (0<\mu \leq 1,0 \leq \varphi \leq 2 \pi),
\end{array}
$$

where $\tau_{1}$ is the total optical thickness of the atmosphere.

It is another surprise that in the mathematical treatment of the above equation Chandraseinhar has succeeded to reduce the solution of the integro-differential equation to the solution of a basic set of integral equations using his principle of invariance. The basic set of integral equations are written as follows:

$$
\begin{gathered}
X(\mu)=1+\mu \int_{0}^{1} \frac{T\left(\mu^{\prime}\right)}{\mu+\mu^{\prime}}\left[X(\mu) X\left(\mu^{\prime}\right)-Y(\mu) Y\left(\mu^{\prime}\right)\right] d \mu^{\prime}, \\
Y(\mu)=e^{-\tau_{1 / \mu}}+\mu \int_{0}^{1} \frac{\Psi\left(\mu^{\prime}\right)}{\mu-\mu^{\prime}}\left[Y(\mu) X\left(\mu^{\prime}\right)-X(\mu) Y\left(\mu^{\prime}\right)\right] d \mu^{\prime},
\end{gathered}
$$

where the characteristic function $\Psi(\mu)$ is an even polynomial of $\mu$ and there are four cases written as follows:

$$
\begin{aligned}
\Psi_{l}(\mu) & =\frac{3}{4}\left(1-\mu^{2}\right), \\
\Psi_{r}(\mu) & =\frac{3}{8}\left(1-\mu^{2}\right), \\
\Psi^{(1)}(\mu) & =\frac{3}{8}\left(1-\mu^{2}\right)\left(1+2 \mu^{2}\right), \\
\Psi^{(2)}(\mu) & =\frac{3}{16}\left(1+\mu^{2}\right)^{2} .
\end{aligned}
$$

Thus each of the corresponding functions $X_{l}(\mu), Y_{l}(\mu), X_{r}(\mu), Y_{r}(\mu), X^{(1)}(\mu)$, $Y^{(1)}(\mu), X^{(2)}(\mu)$ and $Y^{(2)}(\mu)$ can be determined.

Now the final formulae of the intensity on the ground can be expressed as 
the sum of three terms like the formulas of scattering matrix (3), (4), (5), (6) and (7), the azimuth independent terms, the terms including $\left(\varphi^{\prime}-\varphi\right)$ and the terms including $2\left(\varphi^{\prime}-\varphi\right)$. Of the three terms the azimuth independent is the most complicated and in order to express these, further eight auxiliary functions of $\mu$, that is, $\xi, \eta, \sigma, \theta, \varphi, \chi, \phi$ and $\zeta$ are needed which are related with $X_{l}, Y_{l}, X_{r}$ and $Y_{r}$ in a rather complicated way and we shall not write here the equations. (See "Radiative Transfer" p. 269).

Using the twelve functions $\xi, \eta, \sigma, 0, \varphi, \zeta, X^{(1)}, Y^{(1)}, X^{(2)}$ and $Y^{(2)}$ the final formulae become as follows:

$$
\begin{aligned}
& I_{l}\left(\tau_{1} ;-\mu, \varphi ; \mu_{0}, \varphi_{0}\right)=\frac{3}{32}\left[\left\{\phi\left(\mu_{0}\right)+\chi\left(\mu_{0}\right)\right\} \xi(\mu)+2\left\{\phi\left(\mu_{0}\right)+\zeta\left(\mu_{0}\right)\right\} \eta(\mu)\right. \\
& \quad-\left\{\xi\left(\mu_{0}\right)+\sigma\left(\mu_{0}\right)\right\} \phi(\mu)-2\left\{\theta\left(\mu_{0}\right)+\eta\left(\mu_{0}\right)\right\} \phi(\mu)+4 \mu \mu_{0}\left(1-\mu^{2}\right)^{\frac{1}{2}}\left(1-\mu_{0}^{2}\right)^{\frac{1}{2}} \\
& \quad \times\left\{X^{(1)}\left(\mu_{0}\right) Y^{(1)}(\mu)-Y^{(1)}\left(\mu_{0}\right) X^{(1)}(\mu)\right\} \cos \left(\varphi_{0}-\varphi\right)-\mu^{2}\left(1-\mu_{0}^{2}\right)\left\{X^{(2)}\left(\mu_{0}\right) Y^{(2)}(\mu)\right. \\
& \left.\left.-Y^{(2)}\left(\mu_{0}\right) X^{(2)}(\mu)\right\} \cos 2\left(\varphi_{0}-\varphi\right)\right] \frac{F \mu_{0}}{\mu-\mu_{0}}, \\
& I_{r}\left(\tau_{1} ;-\mu, \varphi ; \mu_{0}, \varphi_{0}\right)=\frac{3}{32}\left[\left\{\phi\left(\mu_{0}\right)+\chi\left(\mu_{0}\right)\right\} \sigma(\mu)+2\left\{\phi\left(\mu_{0}\right)+\zeta\left(\mu_{0}\right)\right\} \theta(\mu)\right. \\
& -\left\{\xi\left(\mu_{0}\right)+\sigma\left(\mu_{0}\right)\right\} \chi(\mu)-2\left\{\theta\left(\mu_{0}\right)+\eta\left(\mu_{0}\right)\right\} \zeta(\mu)+\left(1-\mu_{0}^{2}\right)\left\{X^{(2)}\left(\mu_{0}\right) Y^{(2)}(\mu)\right. \\
& \left.\left.-Y^{(2)}\left(\mu_{0}\right) X^{(2)}(\mu)\right\} \cos 2\left(\varphi_{0}-\varphi\right)\right] \frac{F \mu_{0}}{\mu-\mu_{0}}, \\
& U\left(\tau_{1} ;-\mu, \varphi ; \mu_{0}, \varphi_{0}\right)=\frac{3}{16}\left[2 ( 1 - \mu ^ { 2 } ) ^ { \frac { 1 } { 2 } } ( 1 - \mu _ { 0 } ^ { 2 } ) ^ { \frac { 1 } { 2 } } \mu _ { 0 } \left\{X^{(1)}\left(\mu_{0}\right) Y^{(1)}(\mu)\right.\right. \\
& \left.-Y^{(1)}\left(\mu_{0}\right) X^{(1)}(\mu)\right\} \sin \left(\varphi_{0}-\varphi\right)-\mu\left(1-\mu_{0}^{2}\right)\left\{X^{(2)}\left(\mu_{0}\right) Y^{(2)}(\mu)\right. \\
& \left.\left.-X^{(2)}\left(\mu_{0}\right) X^{(2)}(\mu)\right\} \sin 2\left(\varphi_{0}-\varphi\right)\right] \frac{F \mu_{0}}{\mu-\mu_{0}},
\end{aligned}
$$

where $\boldsymbol{F}=\left(\frac{1}{2} F, \frac{1}{2} F, 0\right)$, assuming that the incident light is unpolarized.

\section{On the numerical calculation of the formulae}

The fundamental problem is the numerical solution of the integral equations of (9) and (10). In "Radiative Transfer" pp. 202 206 formulae of the corrected second approximation are described and the neccessary numerical tables are given in the Astrophysical Journal[12], [13]. However the available range of this computation is restricted to comparatively small values of $\tau_{1}$, (at most 0.2 or so) and in order to do the calculation in the ultraviolet range of wave-length $\left(\tau_{1}=0.5-1.0\right)$, more accurate values of $X(\mu)$ and $Y(\mu)$ are neccessary, extending further the approximation of sucessive iteration. Recently this has been accomplished by ChANDRASEKHAR himself with the use of an electronic computor and the numerical tables of the above twelve functions when $\tau_{1}=0.05,0.10,0.15,0.20,0.25,0.50$ and 1.0 have been published[8]. The present author was happy enough to be sent this table by Prof. Chandraserhar and was able to carry through the calculation by the formulae (12).

As to the condition of calculation the values of $I_{l}+I_{\text {r }}$ when $\pi F=1 ; \mu_{0}=0.86$ and $0.5 ; \mu=1.0,0.98,0.94,0.86,0.76,0.64$ and $0.5 ; \varphi_{0}-\varphi=\pi$ and $\tau_{1}=0.2,0.25,0.5$ and 1.0 were computed so that we can obtain the intensity from varying direc- 
tions each seperating by about ten degrees of zenith angle in the sun's opposite vertical plane in the wave-length ranging from about $\lambda 4660 \AA$ to $\lambda 3115 \AA$. The results are given in Table 1.

Table 1. Intensities of sky light calculated by the method of radiative equilibrium (where $F=1 / \pi$ ).

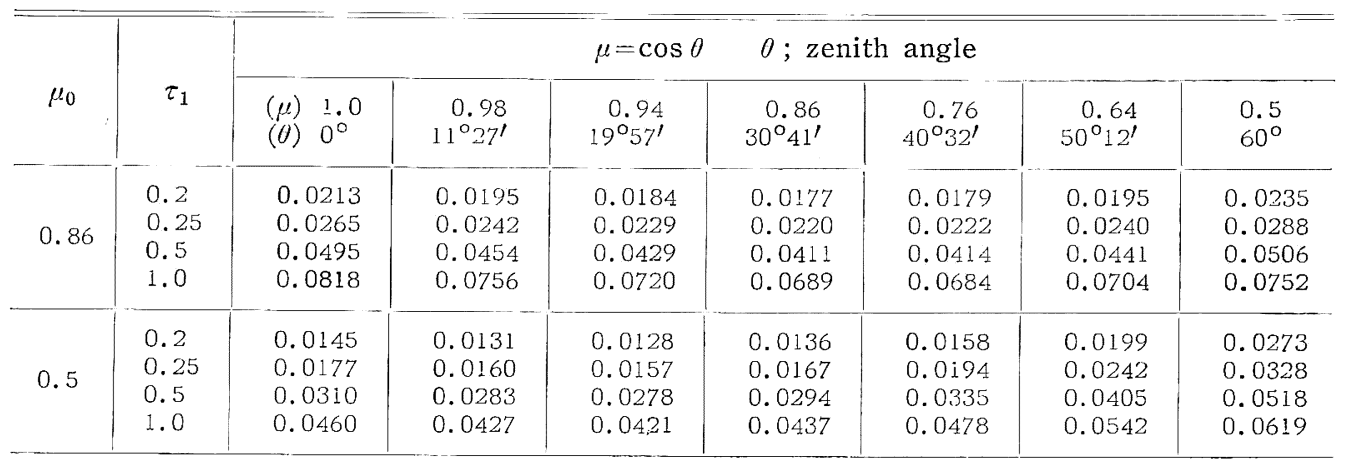

\section{The method of direct integration}

This method does not start from the above integro-differential equation, but from the integral equation of the type introduced by L. V. King[14].* Using the same notations as in the previous section, it is written down as follows:

$$
\begin{gathered}
\boldsymbol{I}(\tau, \mu, \varphi)=\frac{1}{4} e^{-\tau / \mu_{0}} \boldsymbol{P}\left(\mu, \varphi ;-\mu_{0}, \varphi_{0}\right) \boldsymbol{F}+\frac{1}{4 \pi} \int_{-1}^{+1} \int_{0}^{2 \pi} \int_{0}^{\tau_{1}} \boldsymbol{P}\left(\mu, \varphi ; \mu^{\prime}, \varphi^{\prime}\right) \\
\times \boldsymbol{I}\left(\tau^{\prime}, \mu^{\prime}, \varphi^{\prime}\right) e^{-\int_{\tau}^{\tau} \frac{d \tau^{\prime}}{\mu^{\prime}}} d \mu^{\prime} d \varphi^{\prime} \frac{d \tau^{\prime}}{\mu^{\prime}}
\end{gathered}
$$

We are going to solve this equation approximately. Consider the case when the thickness of the atmosphere is comparatively small and the incident direct radiation is predominant everywhere, then it follows that the first term of the right hand side of the equation is the principal one. Therefore we can obtain the approximate value of $\boldsymbol{I}(\tau, \mu, \varphi)$ by evaluating the terms of the right hand side where we replace $\mathbb{I}\left(\tau^{\prime}, \mu^{\prime}, \varphi^{\prime}\right)$ of the second term by the first term itself. This is merely the direct integration of the primary and secondary scattered light. This method was first formulated by HAMMAD and CHAPMAN[1] and later developed and corrected by HAMMAD[2] and also by this author[4]. Here we shall rewrite the result of calculation in Table 2 which was previously pulished by this author [16].

It should be noted that the scattering law adopted there was RAYLEIGH's law, as in the case of CHANDRAsekHAR. However, owing to a quite general and abstract expression in the case of radiative equilibrium it is not clear whether the two scattering laws agree or not. Now it can be seen in the following way.

Let, in the rectangular coordinate $(x, y, z)$, a plane polarized light beam of unit intensity oscillating in $z$ direction propagate along $x$ axis and be scattered

* It was proved by JACKSON[15] that the solution of the equation of radiative equilibrium of the type of differential equation satisfied the integral equation of this type. 
Table 2. Intensities of sky light due to primary and secondary scattering calculated from direct integration.

\begin{tabular}{c|c|c|c|c|c|c|c|c}
\hline$Z$ & $\tau_{1}$ & $0^{\circ}$ & $10^{\circ}$ & $20^{\circ}$ & $30^{\circ}$ & $40^{\circ}$ & $50^{\circ}$ & $60^{\circ}$ \\
\hline \multirow{3}{*}{$30^{\circ}$} & 0.2 & 0.0205 & 0.0189 & 0.0176 & 0.0169 & 0.0169 & 0.0182 & 0.0219 \\
& 0.4 & 0.0363 & 0.0334 & 0.0310 & 0.0295 & 0.0292 & 0.0308 & 0.0357 \\
& 0.6 & 0.0468 & 0.0431 & 0.0399 & 0.0375 & 0.0365 & 0.0381 & 0.0425 \\
& 0.8 & 0.0530 & 0.0487 & 0.0449 & 0.0421 & 0.0406 & 0.0412 & 0.0444 \\
& 1.0 & 0.0557 & 0.0512 & 0.0473 & 0.0440 & 0.0421 & 0.0417 & 0.0431 \\
\hline \multirow{3}{*}{$60^{\circ}$} & 0.2 & 0.0136 & 0.0124 & 0.0120 & 0.0126 & 0.0146 & 0.0184 & 0.0253 \\
& 0.4 & 0.0225 & 0.0205 & 0.0198 & 0.0206 & 0.0233 & 0.0287 & 0.0378 \\
& 0.6 & 0.0272 & 0.0248 & 0.0238 & 0.0246 & 0.0274 & 0.0327 & 0.0412 \\
& 0.8 & 0.0287 & 0.0261 & 0.0249 & 0.0255 & 0.0279 & 0.0327 & 0.0391 \\
& 1.0 & 0.0283 & 0.0257 & 0.0246 & 0.0244 & 0.0262 & 0.0298 & 0.0346 \\
\hline
\end{tabular}

at the origin in the direction of $\alpha, \beta, \gamma$ of the direction cosines (See Fig. 1). Then how much is the intensity of the scattered light and in what direction the scattered light will oscillate?

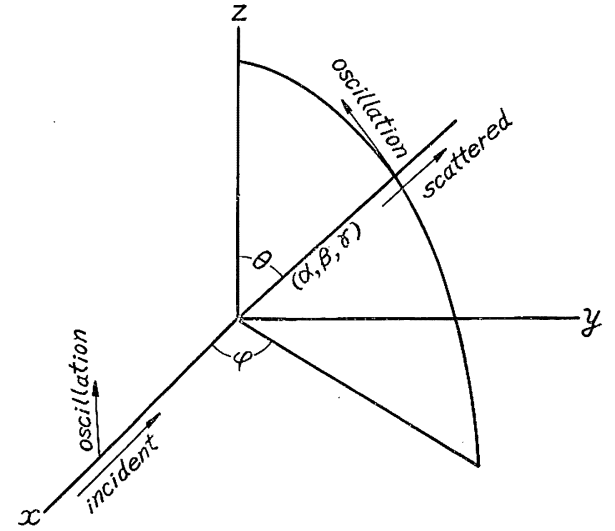

Fig. 1. An illustrative diagram of light scattering.

According to the discussion described previously concerning the theory of direct integration, the intensity of the scattered light per unit solid angle is $\frac{3 \sigma}{8 \pi}\left(1-\gamma^{2}\right)$ and the direction cosine of the direction of oscillation of the scattered light is $\left(\frac{\alpha \gamma}{\sqrt{1-\gamma^{2}}}\right.$, $\left.\frac{\beta \gamma}{\sqrt{1-\gamma^{2}}}, \frac{-\left(\alpha^{2}+\beta^{2}\right)}{\sqrt{1-\gamma^{2}}}\right)$. Now let us write this using the expression of vector and matrix of the previous section.

First the intensity of the incident light is $\mathbb{I}=(1,0,0)$ where we take $l$ direction the same as that of $z$ axis. And as to the direction of incident and scattered light the following relations are easily seen to hold: $\mu^{\prime}=\varphi^{\prime}=0, \quad \mu=\gamma, \cos \varphi=\frac{\alpha}{\sqrt{1-\gamma^{2}}}$ $\sin \varphi=\frac{\beta}{\sqrt{1-\gamma^{2}}}$. Using these relations and remembering that $\boldsymbol{I}_{s}=\frac{\sigma}{4 \pi} \boldsymbol{P I}$, where $\boldsymbol{I}_{s}$ and $\boldsymbol{I}$ are scattered and incident light respectively, we can get from (3), (4), (5), (6) and (7) $\boldsymbol{I}_{s}=\left(\frac{2 \rho}{8 \pi}\left(1-\boldsymbol{\gamma}^{2}\right), 0,0\right)$. Then the intensity is quite the same as what was written above. As the components of $\boldsymbol{I}_{s}$ are zero except for $l$-component, the direction of oscillation of scattered light lies in the meridian plane. After calculating the direction cosines we can get the same result as the previous one $\left(\frac{\alpha \gamma}{\sqrt{1-\gamma^{2}}}, \frac{\beta \gamma}{\sqrt{1-\gamma^{2}}}, \frac{-\left(\alpha^{2}+\beta^{2}\right)}{\sqrt{1-\gamma^{2}}}\right)$.

\section{Observation of the distribution of ultraviolet sky radiation}

Observations of this kind have been done up to now by DoRno and Lindholm[17], EcKeL[18], and other, mostly European, researchers, using cadmium photoelectric cells. Consequently the range of wave-length of these measurements were con- 
fined to the shorter region than $3200 \AA$ of sky radiation. And there are few cases as far as the author knows, when the measurement was done simultaneously in different ranges of wave-length of ultraviolet sky radiation. Measurement of the light from the zenith sky is, however, done very accurately using the quartz spectrometer with a view to observing atmospheric ozone. In this case analysis has been so far carried out only on the primary scattered light. But the contribution of the multiply scattered light in the ultraviolet sky radiation is, as we shall see, very important and so there is a very serious requirement for the solution of this subject from this practical view point.

This author has made observation on the distribution of ultraviolet sky radiation in two different ranges of wave-length, one roughly ranging $\lambda \lambda 3500-3700 \AA$ and the other $\lambda \lambda 3100 \sim 3300 \AA$, using a multiplier photo-tube and suitable filters, and some of the results were already published[19]. Here we have given in Table 3 the data of relative intensities in the sun's opposite vertical plane. When the

Table 3. Observed distributions of ultraviolet sky radiation in the sun's opposite vertical plane.

a) $Z=31^{\circ}\left(h=59^{\circ}\right)$ May 15,$1952 ;$ Tokyo

\begin{tabular}{|c|c|c|c|c|c|}
\hline$\lambda(\AA)$ & $0^{\circ}$ & $15^{\circ}$ & $30^{\circ}$ & $45^{\circ}$ & 60 \\
\hline $3100-3300$ & 1.00 & 0.81 & 0.71 & 0.61 & 0.62 \\
\hline $3500-3700$ & 1.00 & 0.78 & 0.70 & 0.71 & 0.76 \\
\hline
\end{tabular}

b) $Z=60^{\circ}\left(h=30^{\circ}\right)$ September 26, 1953; Tokyo

\begin{tabular}{r|c|c|c|c|c|c|c}
\hline$\lambda(\AA)$ & 0 & $10^{\circ}$ & $20^{\circ}$ & $30^{\circ}$ & $40^{\circ}$ & $50^{\circ}$ & $60^{\circ}$ \\
\hline $3100-3300$ & 1.00 & 0.93 & 0.91 & 0.95 & 1.05 & 1.16 & 1.30 \\
$3500-3700$ & 1.00 & 0.91 & 0.91 & 0.95 & 1.10 & 1.29 & 1.54 \\
\hline
\end{tabular}

sun's zenith angle is $30^{\circ}$ and $60^{\circ}$ respectively. Each observation was done in Tokyo on clear days and both measurements of different ranges of wave-length were done almost simultaneously, that is within a few minutes of each other.

\section{Comparison of the theories with observation}

At first we shall give in Fig. 2 the variation of the calculated intensities in the zenith with $\tau_{1}$, showing in what manner the theories, the method of radiative equilibrium and that of direct integration, varies with the variation of the optical thickness of the atmosphere or, what comes to the same thing, with the variation of the wave-length. According to this figure, the difference of the two curves which show the contribution of the scattered light higher than the second does not exceed several percent of the total intensity at both of the sun's zenith angle when $\tau_{1}=0.2(\lambda=4660 \AA)$, but when the wave-length becomes shorter and $\tau_{1}=1.0(\lambda=3115 \AA)$, it amounts to about $30 \%$ at the sun's zenith angle of $30^{\circ}$, and to about $40 \%$ at the sun's zenith angle of $60^{\circ}$.

Now to see what kind of influence these multiply scattered light exerts on the distribution, the data of Tables 1 and 2 are converted to the relative values taking the zenith intensity as a unit and were given in Tables 4 and 5 and then 
these were compared with the observed distribution. As to the wave-length of actual observation the longer region roughly corresponds to $\tau_{1}=0.5$ and the shorter one to $\tau_{1}=1.0$. In Fig. 3 are shown the curves of actual observation with the theoretical curves of radiative equilibrium. To avoid the complexity of the figures the theoretical curves of direct integration were omitted, and the reader is referred to Table 5 for these figures.

First, regarding the curves when $z=60^{\circ}$ Fig. $3 \mathrm{~b}$, we may say that the agreement between theory and observation is quite satisfactory in both ranges of wave-length. On the other hand, as may be seen from Table 5, the theoretical values of direct integration not only disagree with observation, but also they

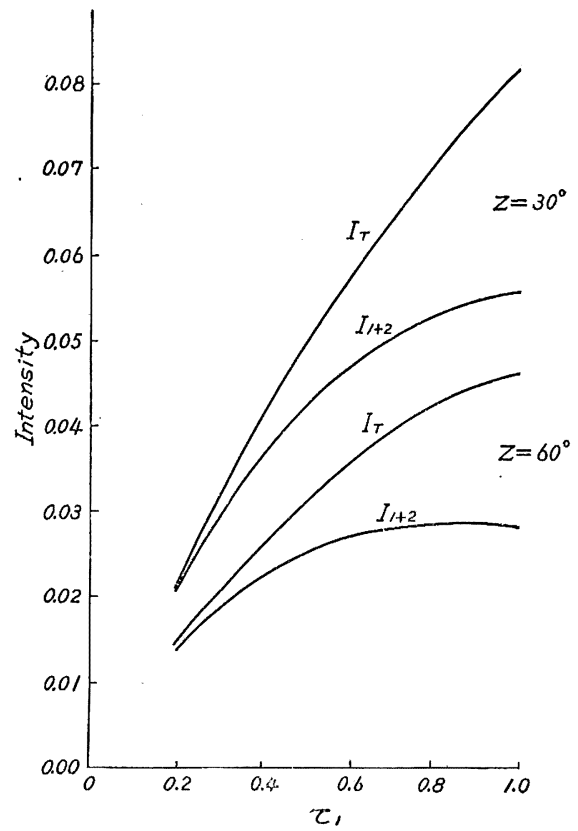

Fig. 2. Intensities of zenith sky light calculated from radiative equilibrium $\left(I_{T}\right)$ and direct integration $\left(I_{1+2}\right)$ with varying $\tau_{1}$, the opitcal thickness of the atmosphere, each at the sun's zenith angle $(\hat{z})$ of $30^{\circ}$ and $60^{\circ}$ respectively. have no possibility of agreement at all. For, while in the actual observation the relative values in the direction of $10^{\circ}$ of the zenith angle increases as the wave-length range of measurement becomes shorter, the theoretical values of Table 5 . do not show this tendency but rather decrease, only the theoretical values of radiative equilibrium of Table 4 showing this tendency.

Next, turning to the distribution when $z=30^{\circ}$ (Fig. 3a), neither of the theoretical values agree with the observed ones. For, the actual curves are always extremely steep and the values of large zenith angles are always very low. But the two curves of different wave-lengths are crossing at about $\theta=40^{\circ}$, and show the abovementioned tendency as seen in the theoretical curves of radiative equilibrium. The reason of the disagrement is presumably because a nonRayleigh scattering has influenced the distribution, which anomalously increasses the intensity near the direction of the sun. This is another problem of interest to be studied in future.

Table 4. Theoretical distribution of ultraviolet sky radiation (from radiative equilibrium).

\begin{tabular}{|c|c|c|c|c|c|c|c|c|}
\hline$\mu_{0}$ & $\mu$ & 1.0 & 0.98 & 0.94 & 0.86 & 0.76 & 0.64 & 0.5 . \\
\hline 0.86 & $\begin{array}{l}0.2 \\
0.25 \\
0.5 \\
1.0\end{array}$ & $\begin{array}{l}1.000 \\
1.000 \\
1.000 \\
1.000\end{array}$ & $\begin{array}{l}0.912 \\
0.912 \\
0.917 \\
0.925\end{array}$ & $\begin{array}{l}0.860 \\
0.863 \\
0.866 \\
0.880\end{array}$ & $\begin{array}{l}0.828 \\
0.828 \\
0.830 \\
0.843\end{array}$ & $\begin{array}{l}0.838 \\
0.838 \\
0.836 \\
0.836\end{array}$ & $\begin{array}{l}0.912 \\
0.907 \\
0.891 \\
0.861\end{array}$ & $\begin{array}{l}1.100 \\
1.086 \\
1.022^{\circ} \\
0.919\end{array}$ \\
\hline 0.5 & $\begin{array}{l}0.2 \\
0.25 \\
0.5 \\
1.0\end{array}$ & $\begin{array}{l}1.000 \\
1.000 \\
1.000 \\
1.000\end{array}$ & $\begin{array}{l}0.905 \\
0.907 \\
0.915 \\
0.929\end{array}$ & $\begin{array}{l}0.887 \\
0.890 \\
0.899 \\
0.915\end{array}$ & $\begin{array}{l}0.943 \\
0.946 \\
0.951 \\
0.950\end{array}$ & $\begin{array}{l}1.095 \\
1.096 \\
1.082 \\
1.039\end{array}$ & $\begin{array}{l}1.378 \\
1.371 \\
1.307 \\
1.178\end{array}$ & $\begin{array}{l}1.891 \\
1.855 \\
1.674 \\
1.346\end{array}$ \\
\hline
\end{tabular}


Table 5. Theoretical distribution of ultraviolet sky radiation (from direct integration).

\begin{tabular}{|c|c|c|c|c|c|c|c|c|}
\hline$Z$ & $\tau_{1}$ & $0^{\circ}$ & $10^{\circ}$ & $20^{\circ}$ & $30^{\circ}$ & $40^{\circ}$ & $50^{\circ}$ & $60^{\circ}$ \\
\hline $30^{\circ}$ & $\begin{array}{l}0.2 \\
0.4 \\
0.6 \\
0.8 \\
1.0\end{array}$ & $\begin{array}{l}1.000 \\
1.000 \\
1.000 \\
1.000 \\
1.000\end{array}$ & $\begin{array}{l}0.922 \\
0.922 \\
0.921 \\
0.919 \\
0.918\end{array}$ & $\begin{array}{l}0.856 \\
0.855 \\
0.852 \\
0.848 \\
0.845\end{array}$ & $\begin{array}{l}0.820 \\
0.813 \\
0.802 \\
0.794 \\
0.790\end{array}$ & $\begin{array}{l}0.823 \\
0.805 \\
0.780 \\
0.766 \\
0.756\end{array}$ & $\begin{array}{l}0.877 \\
0.850 \\
0.814 \\
0.778 \\
0.748\end{array}$ & $\begin{array}{l}1.066 \\
0.983 \\
0.909 \\
0.837 \\
0.772\end{array}$ \\
\hline $60^{\circ}$ & $\begin{array}{l}0.2 \\
0.4 \\
0.6 \\
0.8 \\
1.0\end{array}$ & $\begin{array}{l}1.000 \\
1.000 \\
1.000 \\
1.000 \\
1.000\end{array}$ & $\begin{array}{l}0.910 \\
0.910 \\
0.910 \\
0.909 \\
0.909\end{array}$ & $\begin{array}{l}0.880 \\
0.877 \\
0.876 \\
0.869 \\
0.869\end{array}$ & $\begin{array}{l}0.926 \\
0.914 \\
0.904 \\
0.888 \\
0.864\end{array}$ & $\begin{array}{l}1.066 \\
1.036 \\
1.006 \\
0.973 \\
0.921\end{array}$ & $\begin{array}{l}\text { 1. } 345 \\
1.272 \\
1.204 \\
1.140 \\
1.053\end{array}$ & $\begin{array}{l}1.851 \\
1.675 \\
1.515 \\
1.361 \\
1.224\end{array}$ \\
\hline
\end{tabular}
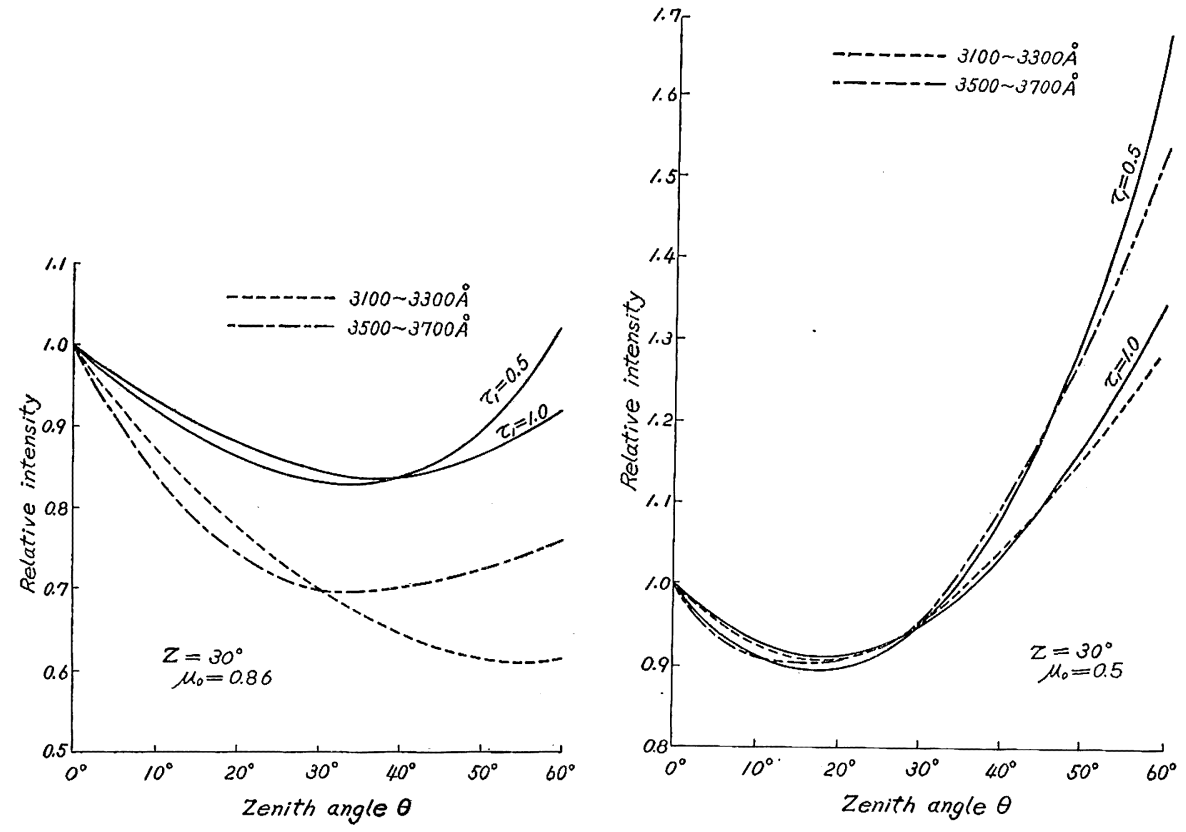

Fig. 3. Distributions of ultraviolet sky radiation in the sun's opposite vertical plane, from theory (radiative equilibrium) and observation.

\section{The simple method of evaluation of the scattered light of higher orders}

Recently J. Lenoble[20] and F. Bernhardt[21] have calculated the total intensity of sky radiation where they estimated the scattered intensity of higher orders by linearly extrapolating the decreasing ratio of successive orders of scattering and evaluated the scattered intensity of all orders as a summation of an infinite geometrical series. However, by that process of calculation LENOBLE has simplified the directional property of light scattering as spherical symmetry and BERNHARDT has adopted the so-called RAYLEIGH's phase function as a fundamental scattering law, neglecting the effect of polarization. And very recently DEIRMENDJian and SeKERA[11] have calculated the scattered radiation from the whole sky using the 
Table 6. Intensities calculated approximately and their errors.

\begin{tabular}{|c|c|c|c|c|c|c|c|c|c|}
\hline$Z$ & $\tau_{1}$ & $\theta$ & $0^{\circ}$ & $10^{\circ}$ & $20^{\circ}$ & $30^{\circ}$ & $40^{\circ}$ & $50^{\circ}$ & $60^{\circ}$ \\
\hline & 0.2 & $\begin{array}{l}I_{1} \\
a \\
I_{a p p} \\
\text { error } \quad(\%)\end{array}$ & $\begin{array}{l}0.0169 \\
0.219 \\
0.0216 \\
+1.4\end{array}$ & $\begin{aligned} & 0.0155 \\
& 0.219 \\
& 0.0199 \\
+ & 2.0\end{aligned}$ & $\begin{array}{r}0.9 \\
0.2 \\
0.0 \\
+0.5\end{array}$ & $\begin{array}{l}0.0137 \\
0.234 \\
0.0179 \\
+1.1\end{array}$ & $\begin{aligned} & 0.0136 \\
& 0.243 \\
& 0.0180 \\
&+ 0.6\end{aligned}$ & $\begin{array}{l}0.0146 \\
0.247 \\
0.0194 \\
-0.5\end{array}$ & $\begin{array}{l}0.0174 \\
0.247 \\
0.0231 \\
-1.7\end{array}$ \\
\hline & 1.0 & $\begin{array}{l}I_{1} \\
a \\
I_{a p p} \\
\text { error }(\%)\end{array}$ & $\begin{array}{l}0.0356 \\
0.567 \\
0.0822 \\
+0.5\end{array}$ & $\begin{aligned} & 0.0325 \\
& 0.575 \\
& 0.0765 \\
+ & 1.2\end{aligned}$ & $\begin{array}{l}0.0296 \\
0.598 \\
0.0736 \\
+2.2\end{array}$ & $\begin{array}{l}0.0272 \\
0.621 \\
0.0718 \\
+4.2\end{array}$ & $\begin{array}{l}0.0254 \\
0.657 \\
0.0741 \\
+8.3\end{array}$ & $\begin{array}{l}0.0248 \\
0.677 \\
0.0768 \\
+9.1\end{array}$ & $\begin{aligned} & 0.0254 \\
& 0.697 \\
& 0.0838 \\
&+11.3\end{aligned}$ \\
\hline \multirow{2}{*}{$60^{\circ}$} & 0.2 & $\begin{array}{l}I_{1} \\
a \\
I_{a p p} \\
\text { error }(\%)\end{array}$ & $\begin{array}{l}0.234 \\
0.0145 \\
0\end{array}$ & $\begin{array}{l}0.0100 \\
0.240 \\
0.0132 \\
+0.7\end{array}$ & $\begin{aligned} & 0.0097 \\
& 0.247 \\
& 0.0129 \\
+ & 0.8\end{aligned}$ & $\begin{array}{l}0.257 \\
0.0136 \\
0\end{array}$ & $\begin{array}{l}0.0115 \\
0.261 \\
0.0156 \\
-1.3\end{array}$ & $\begin{array}{l}0.0145 \\
0.262 \\
0.0196 \\
-2.0\end{array}$ & $\begin{aligned} & 0.0200 \\
& 0.265 \\
& 0.0272 \\
- & 0.4\end{aligned}$ \\
\hline & 1.0 & $\begin{array}{l}I_{1} \\
a \\
I_{a p p} \\
\text { error } \quad(\%)\end{array}$ & $\begin{array}{l}0.0174 \\
0.626 \\
0.0465 \\
+1.3\end{array}$ & $\begin{aligned} & 0.0156 \\
& 0.647 \\
& 0.0442 \\
+ & 3.5\end{aligned}$ & $\begin{array}{l}0.0149 \\
0.651 \\
0.0427 \\
+1.4\end{array}$ & $\begin{aligned} & 0.0147 \\
& 0.667 \\
& 0.0441 \\
&+ 0.9\end{aligned}$ & $\begin{aligned} & 0.0157 \\
& 0.669 \\
& 0.0474 \\
&-0.8\end{aligned}$ & $\begin{aligned} & 0.0177 \\
& 0.684 \\
& 0.0560 \\
&+ 3.3\end{aligned}$ & $\begin{aligned} & 0.0202 \\
& 0.713 \\
& 0.0704 \\
+ & 13.7\end{aligned}$ \\
\hline
\end{tabular}

where $a=I_{2} / I_{1}, I_{a p p}=I_{1} /(1-a)$

error; $\left(I_{a p p}-I_{T}\right) / I_{T} \times 100$

$I_{1}$; Intensity of primary scattering

$I_{2}$; Intensity of secondary scattering

$I_{T}$; Exact value of intensity calculated from radiative equilibrium

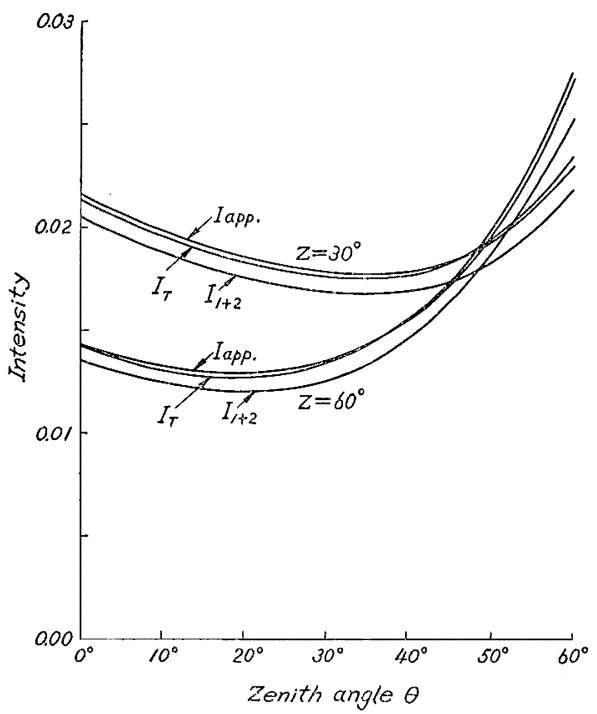

(a) $\tau=0.2$

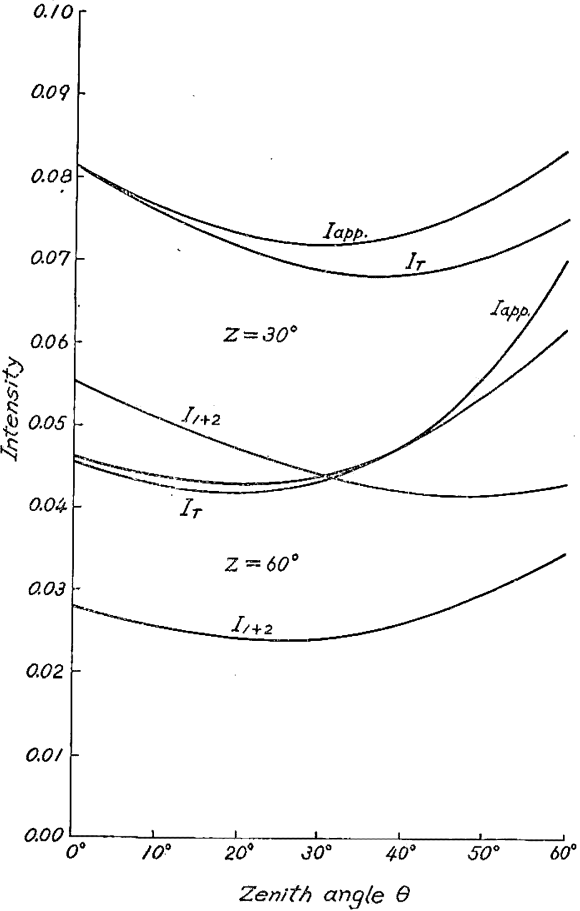

(b) $\tau=1.0$

Fig. 4. Theoretical intensities calculated from radiative equilibrium $\left(I_{T}\right)$, from direct integration $\left(I_{1+2}\right)$ and from approximation $\left(I_{a p p .}\right)$. 
method of radiative equilibrium and compared with BERNHARDT's results. It was shown that BERNHARDT's results gave a marked deviation, greater than $20 \%$, from that of Deirmendian and Sekera even in the visible range of wave-lengths. Now it was already pointed out by the present author[4] that the effect of polarization on the intensity of secondary scattering is not at all small and it will be worth while to examine whether this principle of linear extrapolation is allowable or not on the basis of correct secondary scattering. To do this, using the calculated data of the intensities of primary and secondary scattering which were already published, total intensities of the scattered light of all orders were compared with those of radiative equilibrium. The results when $\tau_{1}=0.2$ and 1.0 are given in Table 6. In Fig. 4 a) and b) are drawn the curves of the distribution obtained from radiative equilibrium, direct integration and simplified approximation respectively. We can see in these figures that when $\tau_{1}=0.2$, the results of simplified approximation agree with the correct ones within the error of a few percent at any direction and at both sun's zenith angle, whereas when $\tau_{1}=1.0$, those of simplified approximation give too large values of more than $10 \%$ especially in the direction of large zenith angles at both of the sun's altitude.

\section{Two different theories of polarization of the sunlit sky}

It is on the sky light polarization[7] that the theory of CHANDraserhar found its practical application and achieved a great success for the first time. The light from the sky is generally partially polarized but in the sun's vertical plane there have been early known three directions, that is, BABINET, BREwSTER and ARAGo neutral points where sky light is not polarized. These points are connected with the point outside of the sun's vertical plane where the plane of polarization of sky light is $45^{\circ}$ inclined with its meridian plane, then composing neutral lines of a form called the lemniscate of Busch. This pattern of neutral line shows a characteristic variation with the change of sun's altitude, according to the very comprehensive observation of $\mathrm{C}$. DoRno[22]. These phenomena were supposed qualitatively to be the effect of secondary and higher order scattering but no satisfactory quantitative theory had been given about them until at last in 1951 ChANDRASEKARA's theory gave a surprisingly clear explanation to these phenomena.

Thus on account of the splendid success of this theory of radiative equilibrium, the theory of direct integration seemed to have lost its effectiveness and interest. But in 1953, Hammad [3] who had most earnestly been engaged in this branch of research, similarly succeeded to explain the neutral point by the theory of direct integration, and besides taking into account of the anisotropy of air molecules he succeded to explain quantitatively the diurnal variation of the degree of polarization of the zenith scattered light which was observed by J. J. Titchanowsky[23], as such a theoretical valus of the maximum polarization is 0.78 for the observed value of 0.77 . For this latter problem ChANDRASEKHAR tried to explain by considering the effect of ground reflexion of the albedo 0.25 , with the theoretical value of 0.9 and thus with rather poor agreement with observation. Consequently, now, it might be presumed that the calculation carried out by CHANDRASEKHAR contains only the secondary scattering. According to the above results of calculation of this author (see Fig. 1 or Fig. 4a) this supposition is clearly confirmed, because 
the difference of the calculated intensities between the two different methods does not exceed, at most, several percent when $\tau_{1}=0.2 \quad(\lambda=4660 \AA)$ and this must certainly decrease to much less for the range of longer wave-length than this.

Thus in the visible range of wave-length the method of direct integration is to some extent as effective as that of radiative equlibrium and moreover it has a merit of simplicity which makes it easy to introduce the complexity of the atmosphere such as non-Rayleigh scattering or absorption layer and so on.

But nevertheless the method of radiative equilibrium is most important for its extreme generality, which makes it effective in the ultraviolet range of wavelength where the method of direct integration utterly fails, as we have seen.

\section{Summary}

1) The intensities of sky light from varying zenith angles in the sun's vertical plane and on its opposite side were calculated in the ranges of wave-length covering through $\lambda \lambda 4660 \sim 3115 \AA$ by means of two different theories, the one being the radiative equilibrium and the other the direct integration, and the results were compared with observation and with each other. Two kinds of observation were used for comparison, the one being the shorter range of wave-length approximately $\lambda \lambda .3100 \sim 3300 \AA$ and the other the longer one approximately $\lambda \lambda .3500 \sim 3700 \AA$.

2) Regarding the distribution when the sun's zenith angle was $60^{\circ}$, the theory of radiative equilibrium explains the observation almost quantitatively, whereas the theory of direct integration cannot, essentially because the scattered light higher than the second increases the relative intensity near the zenith as the range of wave-length becomes shorter, which is not contained in the latter theory.

3) As to the distribution when the sun's zenith angle is $30^{\circ}$, neither of the theoretical curves agree with observation presumably because the contribution of a non-Rayleigh scattering has made the intensities near the sun anomalously strong.

4) The simple approximate method of linear extrapolation for evaluating scattered light of higher orders was examined. In the visible range of wavelength this gives good results within the error of a few percent provided a correct theory of secondary scattering is used. But in the ultraviolet range it makes an error of more than ten percent, especially in the direction of large zenith angles.

5) The amount of scattered light higher than the second is at most several per cent of the total intensity in the visible range, while in the ultraviolet it becomes as much as $30-40 \%$ of the total intensity. And so far as the intensity in the visible range is concerned the method of direct integration is to some extent equally valid as that of radiative equilibrium. 
Acknowledgement_The author wishes to express his hearty thanks to Dr. H. Hatakeyama, Director of the Meteorological Research Institute, for his kind help and encouragement throughout the series of these studies and also to Dr. Y. Mryake, Chief of the Geochemical Laboratory, for his generous permission of the study. Thanks are also due to Prof. Y. Haginara, Director of the Tokyo Astronomical Observatory, Prof. G. Yамамото of Tohoku University, Department of Geophysics, Prof. S. Chandraseinar of Yerkes Observatory, Chicago University U. S. A. for their kind and valuable advices.

\section{References}

[1] Chapman, S. and Hammad, A., 1939: The Primary and Secondary Scattering of Sunlight in a Plain-stratified Atmosphere of Uniform Composition, Phill. Mag. 28, p. 99.

[2] Hammad, A., 1948 : ibid. II. III. Phill. Mag. 38, p. 527 ,, 1947 : ibid., Ap. J. 108, p. 338.

[ 3 ] Hammad, A., 1953: Calculation of the Polarization of a Sunlit Sky Composed of Pure Air Molecules, J. Opt. Soc. Am. 43, p. 184.

[4] Semriara, K., 1951: Studies of the Distribution of Ultraviolet Sky Radiation (II), Pap. Met. Geophys. 2, p. 158.

[5] Chandraserkhar, S., 1944-48: On the Radiative Equilibrium in a Stellar Atmosphere, I-XXIV, Ap. J. 100-108.

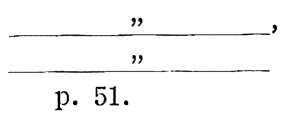
1950: Radiative Transfer, Oxford. and ELBERT, D., 1951: Polarization of the Sunlit Sky, Nature 167, , 1954: The Illumination and Polarization of the Sunlit Sky on Rayleigh Scattering, Trans. Ame. Phil. Soc. New Series, 44, p. 643.

[9] Ashrurn, E. V., 1954: The Effect of Atmospheric Scattering and Ground Reflection upon the Determination of the Height of the Night Airglow, J. Geophys. Res. 59, p. 67.

[10] Deirmendian, D. and Serera, Z., 1953: Quantitative Evaluation of Multiply Scattered and Diffusely Reflected Light in the Direction of a Stellar Source in a Rayleigh Atmosphere. J. Opt. Soc. Am. 43, p. 1158 1954: Global Radiation Resulting from Multiple Scattering in a Rayleigh Atmosphere, Tellus, 6, p. 382.

[12] Chandrasemhar, S. and Breen, F., 1948: On the Radiative Equilibrium of a Stellar Atmosphere XXIV, Appeddix, Ap. J. 108, p. 100.

[13] $\quad, 1949$ : The Functions $\mathrm{G}_{n},{ }_{m}(\tau)$ and $\mathrm{G}_{n}^{\prime}{ }_{n, m}(\tau)$ order $6(\mathrm{~m}=6$ and $\mathrm{m}>\mathrm{n})$ Ap. J. 109, p. 555.

[14] KING, L. V., 1913: On the Scattering and Absorption of Light in Gaseous Media with Application to the Intensity of Sky Radiation, Phil. Trans. Roy. Soc. London, 212, p. 375 .

[15] Jackson, W. H., 1910: The Solution of an Integral Equation occurring in the Theory of Radiation, Bull. Am. Math. Soc. 16, p. 473.

[16] Semifara, K., 1953: Studies of the Distribution of Ultraviolet Sky Radiation (V), Pap. Met. Geophys, 3, p. 277.

[17] Dorno, C. and Lindholm, F., 1929: Helligkeitverteilung über den Himmel im Ultraviolet Met. Z. 46, p. 281.

[18] Ecks,, O., 1934: Die Verteilung der Ultravioletstrahlung über das Himmelsgewölbe, 
Met. Z. 51, p. 180.

[19] Serinara, K., 1954: Observation of the Distribution of Ultraviolet Sky Radiation at Comparatively Low Altitude of the Sun, J. Met. Soc. Japan, Series II, 32, p. 317.

[20] Lenoble, J., 1954: Contribution a l'Etude du Rayonnement Ultraviolet Solaire de sa Diffusion dan l'Atmosphere et de sa Pénétration dan la Mer, Ann. de Geophys. 10, p. 117 .

[21] Bernhardt, F., 1952-53: Die sekundär diffuse Strahlung in einer Rayleigh Atmosphäre, Zs. f. Met. I, 6, p. 257.

", 1953: ibid. Zs. f. Met. II. 7, p. 78.

[22] Dorno, C., 1919: Himmelsheligkeit, Himmelspolarisation und Sonnenintensität in Davos (1911 bis 1918) Met. Z. 36, p. 109.

[23] Tionanowsky, J. J., 1924: Polarisation des Himmelslichtes und Sonnenhöhe Met. Z. 41, p. 352 . 